\title{
Solidification Features of Butadiene and Piperylene Copolymers and Assessment of Thermal-oxidative Stability of Elastic Polyurethane on its Basis
}

\author{
VASILIY P. MEDVEDEV ${ }^{1}$, MARAT A. VANIYEV'1, \\ SAULE A. SAKIBAYEVA ${ }^{2}$ and ANASTASSIYA Y. KOVALEVA²*
}

${ }^{1}$ Volgograd State Technical University, Russian Federation, 400005, Volgograd, Lenin av., 28. ${ }^{2}$ Auezov South Kazakhstan State University, Kazakhstan, 160012, Shymkent, Tauke Khan av., 5. ${ }^{\star}$ Corresponding author E-mail: anastasiya2301@mail.ru http://dx.doi.org/10.13005/ojc/330123

(Received: November 28, 2016; Accepted: January 22, 2017)

\begin{abstract}
The research results given in this article are predetermined by the necessity of research intensification aimed at finding alternatives reactive oligomers for expanding the range of available compositions of such purpose. At the assessment of technics level in the area of binders for synthetic sports surfaces promising use of the above products as reactive oligomers and prepolymers for the realization of free-form molding surfaces was found.
\end{abstract}

Keywords: Oligodiendiole, Polyisocyanate, Chain branching agent, Polydieneurethane, coatings.

\section{INTRODUCTION}

The main problem of SKDP-N (low molecular weight polybutodiene rubber) oligomer macromolecule is its non-homogeneity and low functionality (average 1.5) by hydroxyl groups. Considerable amount of mono- and nonfunctional molecules builds some limitations for getting polyurethanes of a certain qualities. This predetermined the necessity of new development of SKDP-N based elastomers with a technical value by the usage of components excluding all the deficiencies ${ }^{1,2}$.
The performed investigations studied the particularities of forming of netted structure during hydrous SKDP-N oligodiene single stage curing with polyfunctional reagents. Another observation were the influence of chain diversification agent on kinetic parameters of polyaddition ${ }^{3}$. The connection between structure and qualities of cured material is also discovered. The investigations were made about influence of chemical bond network on polydiene urethane microphase condition based on functionally defective oligomer. The possibility of improving qualities of such materials by regulating 
microphase structure was established. In such objects interreaction parameter of Flori-Huggins with toluene solvent is structurally sensitive to supermolecular material coating based on SKDP-N ${ }^{4}$.

\section{MATERIAL AND METHOD}

For the solving of new problems in the field of development and implementation of synthetic coatings it was decided to use oligomers of diene nature with hydroxyl reactive groups: SKDP-N (product of radical copolymerization of butadiene and piperylene in 50:50 proportion with the weight percentage of single-ended hydroxyl groups of $1.1 \%$ ) and PDI-1K (product of copolymerization of divinyl and isoprene in proportion of 80:20 with the weight percentage of single-ended hydroxyl groups of 0,8 $-1,0 \%)^{5}$.

The research was also about oligoesterisocyanate oligodiene-isocyanate semi polymers: SKU-PPT 4503 (product of interreaction of polyoxypropylene triol model «Laprol 4503» with 2.4-toluene diisocyanate having weight percentage of free isocyanate groups $2,5-3,0 \%$, functionality 3 ); semi polymer ADB-17 (product of interreaction of polyoxypropylene glycol with 2.4-toluene diisocyanate having weight percentage of free isocyanate groups $5.9 \%$, functionality 2) and semi polymer $\Omega \Pi-65-2$ (product of interreaction of SKDP-H with 2.4-toluene diisocyanate) and others.

\section{RESULTS AND DISCUSSION}

Below the following results are discussed: the curing of oligomer SKDP-H with equimolar relation of $\mathrm{NCO}$ - and $\mathrm{OH}$-groups $16^{\circ} \mathrm{C}$ with 0.3 weight part of urethane forming dimethylbenzylamine catalyst and $52^{\circ} \mathrm{C}$ degrees without catalyst. It is observed that on anamorphosises of kinetic curves disappearances of NCO-groups on chemical equations of the second order happen on straight sections. It is distinctive for parallel-consistent reactions of functional groups differing by reacting capabilities (Fig 1). At this point increase of chain diversification agent concentration (glycerin) leads to decrease of urethane forming which is connected with the decreased activity of $\mathrm{OH}$-groups of glycerin in comparison with $\mathrm{OH}$-groups of oligodiene. It is confirmed by the increase of relation of observed rate constants of the first and second section of anamorphosis (characterizes relative activity of functional groups) with the increase of triol content.

If there is not chain diversification agent and catalyst the process of urethane forming at $52^{\circ} \mathrm{C}$ is described with one speed constant. For catalyzed composition linearization of kinetic curve with two speed constants is described by the reactive capabilities of NCO-groups.

To clarify the influence of chemical reaction on the curing process the study of viscosity changing

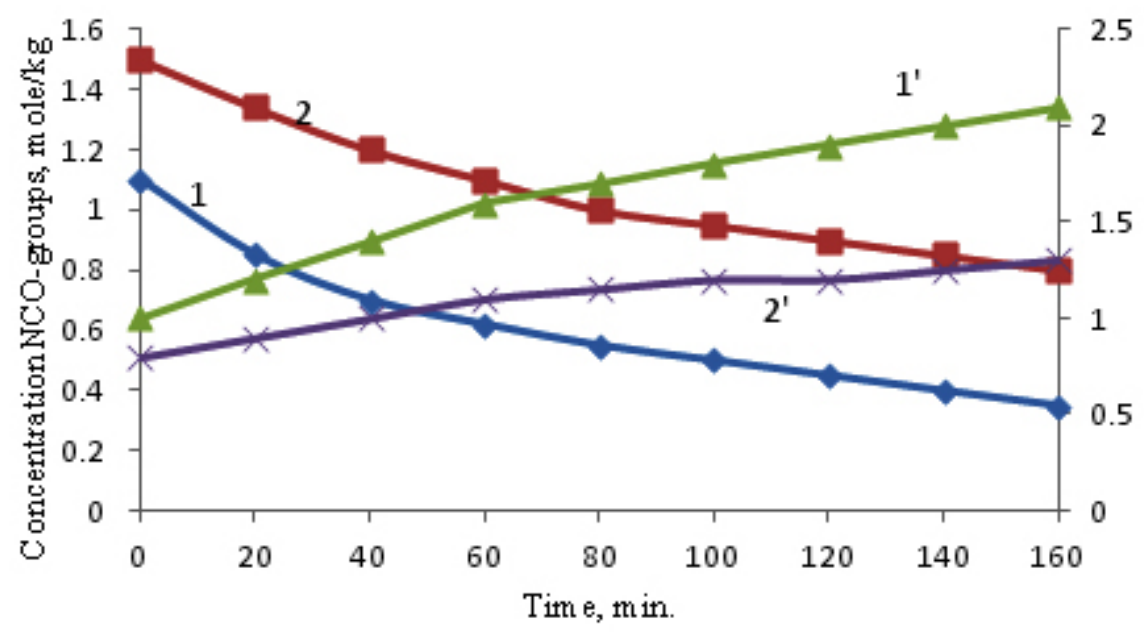

Fig. 1: Cirves of NCO-groups dissapearence $(1,2)$ and their anamorphosises $\left(1^{\prime}, 2^{\prime}\right)$ : triol/ oligodien ratio $0,5(1)$ and $0,9(2)$. Condition: $16^{\circ} \mathrm{C}, 0,3$ weight mass of dimethylbenzylamine 
depending on the changing of $x$ functional groups has been performed. Considering that $\bar{I}_{n}$ of the formed polymer is proportional to the polycondensation degree $P(P=1 / 1-x)$, compound viscosity $\eta$ should change depending from $P$ according to the state law. It was determined that dependence of $h$ from $P$ looks like two straight sections with different tangent angle slopes (Fig. 2).

Viscosity increase rate, which is defined by reaction kinetics, depends on topology development of macromolecular branching. Change of curve slopes is connected with the generation of fluctuating engaging net. This is specified by the growth of both molecular mass and macromolecular branching. Degree index $\mathrm{h}$ in the first approximation characterizes quality and branching degree of formed structures. In this case the increased concentration of urethane forming components increase concentration of active fragments which are capable for donor/acceptor cooperation. The growth of engaging number increases viscosity growth rate with the transformation of functional groups. With the temperature increase of reacting mass the contribution of interchain intercommunications decreases, this leads to less meanings of degree index $\mathrm{h}$. At the some critical reaction level the complete three-dimensional net of chemical and physical bonds is formed. It is determined that gelling process can be satisfactory described with AvramiErofeev equation. Concentration increase of chain branching agent leads to decrease of the specific process speed and increase of Avrami index.

The stated above information shows the particularities of net forming during curing of functionally defective oligomer SKDP-H by polyfunctional reagents. These particularities mean inhomogenity of initial reacting system. Presumably this is dictates by the limited compatibility of SKDP-H with the components of curing system. At the early stage of reaction highly branched macromolecules form which are the first signs of three-dimensional structure. Microheterogenous qualities of the process predetermine forming of gel fraction at the low degrees of functional groups change. It is determined that elastomer net density is determined by triol amount and less dependent on synthesis conditions.
The main result is that the use of polyfunctional curing system allows to produce elastomers on the basis of SKDP-H with lattice density comparable with industrial polyurethane materials. Features of the investigated elastomers structure significantly impact on their physical and mechanical properties. By increasing the ratio of $\mathrm{NCO} / \mathrm{OH}$ elastomers strength properties are improved. When the concentration of effectively cross-linked chains by increasing the content of triol this dependence is extreme. It has a high strength characteristics due to the optimal ratio of the chemical and physical bonds in the overall structure of the grid. As for indicators of material hardness, their values are determined mainly by the content of the polyisocyanate component. Elongation indicators at break with increasing concentrations of cross-linking bonds of elastomers are monotonically decreasing.. Adhesion of the compositions depends on the content of extremely urethane formation components. Maximum adhesion strength with substrates (which is important in the formation of sports coatings) are distinctive for elastomers containing $3-4$ parts by weight glycerol at equimolar ratio NCO- and $\mathrm{OH}$ groups. The highest values of tear resistance are elastomers obtained by $\mathrm{NCO} /$ $\mathrm{OH}>1$.

Thus, in result of the research the ability to control the deformation-strength properties and hardness of elastomers by crosslinking density directional changes is shown. The use of polyfunctional curing agents and chain branching, as well as reasonably selected urethane catalysts, provides compensation for defects oligodienes SKDP-H, related to its molecular heterogeneity.

Taking into account that in the exploitation process hardness increasing and decreasing strength properties and elastic polyurethane elasticity are possible. It is connected with the main bond structuring with á-methylene carbons atom of 1,4-units of oligomer molecule diene fragments, it was necessary to conduct further studies in order to solve the problem of increasing resistance to thermal-oxidative aging of materials based on SKDP-H.

Considering that the operation may increase the hardness and strength properties 
decrease elasticity EPC associated with the structuring backbone with $\alpha$-methylene carbons of 1,4-units diene oligomer molecule fragments, it was necessary to conduct further studies in order to solve the problem of increasing resistance to thermaloxidative aging of materials based on SKDP-H.

With regards to the reactive oligomer the effectiveness of the protective effect of a number of antioxidants of different chemical nature was researched. Stabilizer type selection principle is based on the information about the effectiveness of a particular antioxidant in the composition of the various polymer matrices.
It was taken into account, that firstly, compounds capable of affecting the step of initiating the chain reaction of oxidation (in other words, to increase the induction period prior to the development process), and secondly - a substance that can effectively pick macromolecular chain reaction stage growth .

On the basis of this information, the following compounds were used as a stabilizers: a substituted phenol (Agidol-1), the dihydric phenol (hydroquinone), secondary amine (diafen FF) phosphorous acid ester (triphenyl phosphite) and tris (2,4-di-tert-butylphenyl ) phosphite. The results

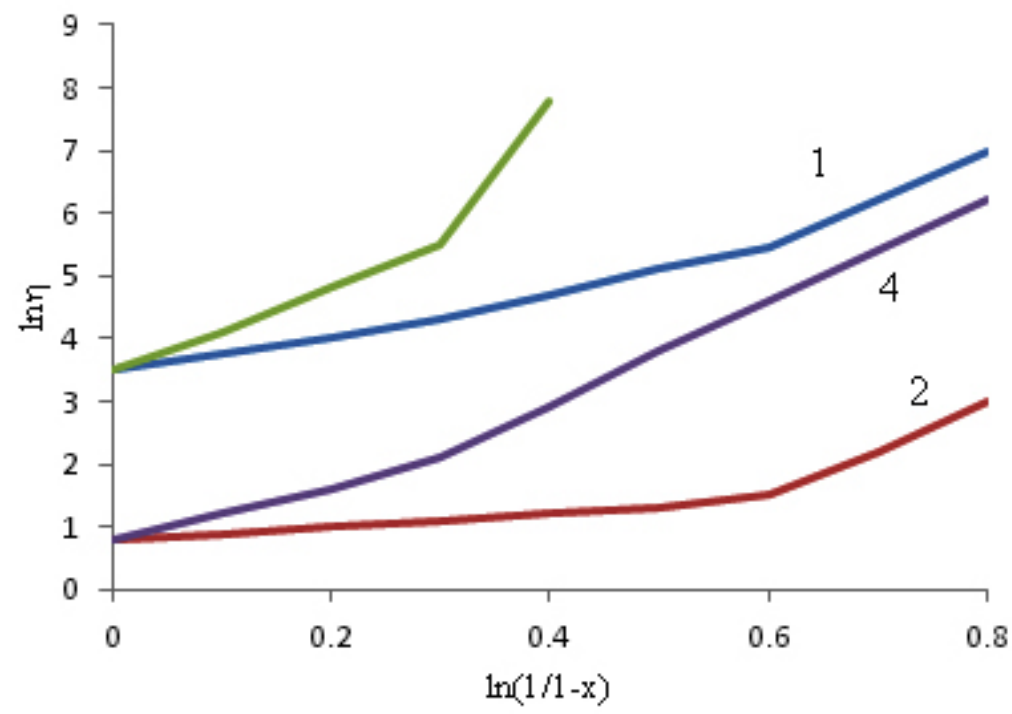

Fig. 2: Viscosity dependence from the polycondensation degree: triol/oligodien ratio $0(1,2) 1,4(3$, 4). Condition: $16^{\circ} \mathrm{C}, 0,3$ weight mass of dimethylbenzylamine $(1,3)$ and $52^{\circ} \mathrm{C}$ without catalyst $(2,4)$.

Table 1: Properties change of elastic polyurethane on the basis of SKDP-H depending on aging time in air medium at $100^{\circ} \mathrm{C}$ and type of stabilizer

\begin{tabular}{|c|c|c|c|c|c|c|c|c|c|c|c|c|}
\hline \multirow{2}{*}{\multicolumn{5}{|c|}{ Solidity, arbitrary unit }} & per & manc & depen & ing on & e tim & $f$ agin & & \\
\hline & & & & & \multicolumn{4}{|c|}{ Tensile strength, MPa } & \multicolumn{4}{|c|}{ Elongation at break, \% } \\
\hline & $\mathbf{O h}$. & $24 \mathrm{~h}$. & $72 \mathrm{~h}$. & $144 \mathrm{~h}$. & $\mathbf{O h}$. & $24 \mathrm{~h}$. & $72 \mathrm{~h}$. & $144 \mathrm{~h}$. & $0 \mathrm{~h}$. & $24 \mathrm{~h}$. & $72 \mathrm{~h}$. & $144 \mathrm{~h}$. \\
\hline 0 & 45 & 56 & 64 & 83 & 1,8 & 0,9 & 0,4 & 0,2 & 130 & 50 & 30 & 20 \\
\hline 1 & 45 & 48 & 49 & 53 & 1,6 & 1,5 & 1,5 & 1,2 & 100 & 100 & 90 & 80 \\
\hline 2 & 43 & 42 & 42 & 42 & 1,5 & 1,5 & 1,4 & 1,4 & 140 & 120 & 120 & 90 \\
\hline 3 & 43 & 47 & 50 & 51 & 1,5 & 1,6 & 1,5 & 1,5 & 180 & 110 & 90 & 80 \\
\hline 4 & 44 & 48 & 80 & - & 1,3 & 1,3 & 0,7 & - & 140 & 80 & 20 & - \\
\hline 5 & 48 & 53 & 44 & 35 & 1,5 & 1,3 & 0,5 & 0,4 & 130 & 150 & 200 & 220 \\
\hline
\end{tabular}


obtained demonstrate the influence of the type of stabilizer at its optimum content on elastomeric properties before and after aging, are presented in table 1.

Samples that do not contain antioxidants, after 72 hours aging dramatically lose strength and flexibility, which is reflected in a sharp fall in elongation values and in increasing their hardness. However, the addition of stabilizers contributes to maintaining the properties at the required level of elastomers. As follows from these data that the greatest protective effect provided by substituted phenol, which is a compound capable of being converted into stable radicals and slow down the development of the linear kinetic chain and the development of a chain reaction of oxidation of the polymer.

Preventive antioxidant, triphenyl phosphite in particular, during the first day has a stabilizing effect on the polyurethane-based elastomers SKDP-H, and are declining significantly after three days. This is probably due to the fact that he becomes the catalyst for the oxidation.

\section{CONCLUSION}

From the data obtained it follows that relatively efficient stabilizer is a phosphorous acid ester, as it combines in a single molecule several active structural fragments.

In compositions of considered type amine nature antioxidant is not efficient (embodiment 5 in table 1). In particular, unlike other additives for polyurethanes, diafenom refilled with increasing aging time there is a decrease in hardness and increase the elongation. Apparently, this can be explained by the destruction of urethane crosslinks resulting aminolysis.

In the result of research it was defined that addition to the composition on the basis SKDP-H stabilizers of defined nature provides the protection of such polydieneurethane elastomers from thermooxidative aging. The most effective were agidol-1 and tris (2,4-di-tert-butylphenyl ) phosphite, added 1 mass. Part to 100 mass part of oligomer.

\section{REFERENCES}

1. 1. Ukrainskaya, S.I.; Medvedev, V.P.; Chapurkin, V.V. Chemistry and technology of elementorganic monomers and polymer materials. 2012.7., 154- 159

2. Medvedev, V.P.; Ukrainskaya, S.I.; Chapurkin, V.V.; Murzin, A.V. Caoutchouc and rubber. 2012, 1, 2-5.

3. Medvedev, V.P.; Ukrainskaya, S.I.; Chapurkin, V.V.; Murzin, A.V. Polyurethane elastomers on the base of NISSO ĐÂ G3000 oligobutandienediol. Glues. Sealants. Technologies. 2012, 12, 11-15.
4. Sergeyeva, L.M.; Lipatove, Y.S.; Binkevich, N.I.. Research of the density of spacing grid and nature of cross-linking bonds in polyurethane elastomers // In book. Synthesis and Physics and Chemistry of polyurethane . 1967, 199

5. Medvedev, V.P.; Vaniyev, M.A.; Sakibayeva, S.A.; Kovaleva, A.Y. Operating Characteristics of Diene-Urethane Elastomers on the base of NISSOG 3000 Oligodienediol. Oriental Journal of Chemistry. 2016, 5, 2363-2370 